\title{
Preparation and Electrocatalytic Study of Myoglobin Biosensor Based on Platinum-Gold-Three Dimensional Graphene Modified Electrode
}

\author{
Bo Shao, Wei Chen, Lijun Yan, Yuhao Huang, Bei Wang, Qingwu Zou, Yongkang Xuan, Wei Sun*, \\ Yanyan Niu*
}

Key Laboratory of Laser Technology and Optoelectronic Functional Materials of Hainan Province, Key Laboratory of Functional Materials and Photoelectrochemistry of Haikou, College of Chemistry and Chemical Engineering, Hainan Normal University, Haikou 571158, China

*E-mail: sunwei@hainnu.edu.cn, niuyanyan171006@hainnu.edu.cn

Received: 5 July 2021 / Accepted: 19 August 2021 / Published: 10 September 2021

\begin{abstract}
An electrochemical biosensor was prepared by using platinum-gold-three dimensional graphene (Pt-Au3DGR) and myoglobin $(\mathrm{Mb})$ with ionic liquid $\mathrm{N}$-hexylpyridinium hexafluorophosphate modified carbon paste electrode (CILE) as the base electrode. The mixed materials of Pt-Au-3DGR and Mb were modified on the electrode to obtain the electrochemical biosensor (Nafion/Mb/Pt-Au-3DGR/CILE). The synthesized Pt-Au-3DGR was characterized by scanning electron microscopy and transmission electron microscopy, which showed a three-dimensional cobweb structure of GR with Pt-Au bimetal successfully loaded on its structure. The biostructure of $\mathrm{Mb}$ was analyzed by UV-Vis spectroscopy and infrared spectroscopy. Direct electrochemistry and electrocatalytic behaviors of this biosensor were tested by cyclic voltammetry and electrochemical impedance spectroscopy, which were further compared with the control group. The results prove that Nafion/Mb/Pt-Au-3DGR/CILE has obvious electrocatalytic functions to trichloroacetic acid (TCA) and sodium nitrite $\left(\mathrm{NaNO}_{2}\right)$. The linear ranges for TCA and $\mathrm{NaNO}_{2}$ are 1.0-30.0 mmol L${ }^{-1}$ and $0.05-0.55 \mathrm{mmol} \mathrm{L}^{-1}$ with the detection limits as $0.33 \mathrm{mmol} \mathrm{L}^{-1}$ and $0.01 \mathrm{mmol} \mathrm{L}^{-1}$, respectively.
\end{abstract}

Keywords: Platinum-gold bimetal; Three-dimensional graphene; Myoglobin; Electrocatalysis

\section{$\underline{\text { FULL TEXT }}$}

(C) 2021 The Authors. Published by ESG (www.electrochemsci.org). This article is an open access article distributed under the terms and conditions of the Creative Commons Attribution license (http://creativecommons.org/licenses/by/4.0/). 